\title{
High-capacity watermarking of high dynamic range images
}

\author{
Emanuele Maiorana* and Patrizio Campisi
}

\begin{abstract}
High dynamic range (HDR) imaging techniques address the need to capture the full range of color and light that the human eyes can perceive in the real world. HDR technology is becoming more and more pervasive. In fact, most of the cameras and smartphones available on the market are capable of capturing HDR images. Among the challenges posed by the spread of this new technology there is the increasing need to design proper techniques to protect the intellectual property of HDR digital media. In this paper, we speculate about the use of watermarking techniques to cope with the peculiarities of HDR media to prevent the misappropriation of HDR images.
\end{abstract}

Keywords: High dynamic range, Data hiding, Watermarking

\section{Introduction}

High dynamic range (HDR) imaging technologies provide a step forward in representing real scenes as they are perceived by the human eye. With respect to traditional imaging techniques capable of acquiring low dynamic range (LDR) images or video, HDR methodologies $[1,2]$ are capable of generating and rendering images and videos with a ratio between the luminance of the lightest and darkest areas far greater than the one provided by standard imaging technologies. It is evident that HDR media are richer in terms of content than their LDR counterparts and therefore much more valuable. It is therefore crucial to provide, since the early stages of the development of such technology, proper tools for protecting the intellectual property of digital HDR media. Data hiding, and more specifically digital watermarking [3-5], has emerged in the last decade as an enabling technology for copyright protection among other possible applications. Roughly speaking, data hiding is the general process by which a discrete information stream, the mark, is merged within media content by imposing imperceptible changes on the original host signal, while allowing the message to be detected or extracted even in the presence of either malevolent or non-intentional attacks. Other requirements, other than the transparency one, can be

*Correspondence: emanuele.maiorana@uniroma3.it

Roma Tre University, Department of Engineering, Section of Applied

Electronics, Via Vito Volterra 62,00146 Rome, Italy needed according to the specific application that is taken into account. Although LDR image or video digital watermarking is a very mature area of research, watermarking in an HDR scenario is still in its infancy and needs to be significantly developed. In this paper, we crystallize the state of the art on HDR image watermarking techniques and the watermarking embedding method proposed by the authors in [6] is more extensively tested using a wider set of images as well as up to date metrics for performance assessment.

A brief overview on digital watermarking is given in Section 2. An extensive and comparative review of the state of the art on HDR image watermarking is given is Section 3. The watermarking method is detailed in Section 4. An extensive set of experimental results is given in Section 5, and conclusions are drawn in Section 6.

\section{Multimedia digital watermarking}

In the past decades, there has been an explosion in the use and distribution of digital multimedia data, essentially driven by the diffusion of the Internet. In this scenario, data hiding and more specifically digital watermarking techniques $[3,4]$ have been proposed to address the ever-growing need to protect the intellectual property of multimedia content (digital still images, 2D and 3D video sequences, text, or audio) in the World Wide Web. Although copyright protection was the very first application of watermarking, different uses have been

\section{Springer}


proposed in the literature. Fingerprinting, copy control, broadcast monitoring, data authentication, multimedia indexing, content-based retrieval applications, medical imaging applications, error concealment, quality assessment, and improved data compression are only a few of the applications where watermarking can be usefully employed. When these techniques are used to preserve the copyright ownership with the purpose of avoiding unauthorized data duplications, the embedded watermark should be detectable. This is required even if malicious attacks or non-malevolent signal processing (i.e., filtering and compression) are applied on the multimedia data. This requirement is known as watermark security. On the other hand, when the watermark is required to be resistant only to non-malicious manipulations, the watermarking techniques are referred to as robust. For some applications, when the robustness requirement is severely required, each attempt of removing the mark should result in irreversible data quality degradation. In some applications, the watermarked host data are intended to undergo a limited number of signal processing operations. Therefore, in the aforementioned scenario, the watermark needs to be robust only to a limited number of set of manipulations, in which case the technique is known as semi-fragile watermarking. On the contrary, when unwanted modifications of the watermarked data affect even the extracted watermark, the embedding scheme is known as fragile. Fragile watermarking can be used to obtain information about the tampering process. In fact, it indicates whether or not the data has been altered and supplies localization information as to where the data was altered. Capacity is another watermarking requirement, referring to the number of bits of information that can be embedded in the original data, which needs to be fulfilled, depending on the specific application. Robustness, imperceptibility, and capacity are requirements hindering each other; therefore, a trade-off driven by the application needs to be considered.

\section{HDR image watermarking: state of the art}

Despite digital watermarking of low dynamic range images having been deeply studied for more than two decades, watermarking of HDR media, both images and video, has not been extensively explored. In fact, a direct transposition of the techniques developed for LDR media is not straightforward since HDR media possess some peculiarities with respect to LDR ones which do not allow a direct transposition of the plethora of watermarking approaches already developed for LDR images and videos to the HDR case. In fact, the specific characteristics of the human visual system (HVS) related to the fruition of HDR media need to be exploited when designing HDR-tailored embedding techniques. Moreover, HDR-specific watermarking methods need to be robust against intentional attacks or signal processing manipulations like the use of tone-mapping operators (TMOs). The use of TMOs is necessary when HDR media need to be experienced using conventional displays in order to generate LDR data retaining as much information as possible from the original objects, while reducing the overall contrast. Therefore, it is highly desirable to design HDR watermarking schemes also robust against TMOs, thus allowing to either recover or detect the embedded mark not only from the marked HDR image but also from its tone-mapped version. In the following, an overview on the state of the art for HDR image watermarking is given. Specifically, in Section 3.1, the 1-bit embedding approaches are detailed, whereas the multi-bit approaches are summarized in Section 3.2.

\subsection{HDR image watermarking: 1-bit embedding}

Watermarking techniques aimed at embedding 1 bit of information imply the detection of the embedded watermark at the receiving side. In [7], two different watermarking methods, both of them splitting the cover HDR image into a host image where to embed the mark and a residual part, are investigated. The first approach employs the $\mu$-law to characterize a generic TMO and applies it to the original HDR image to derive an LDR representation where the watermark can be embedded. The residual part is given by the ratio between the HDR and the LDR images. However, since the range of HDR images may be orders of magnitude greater than the one of LDR images, their ratio will be high in areas with high luminance, where the watermark will be therefore very strong. The second approach decomposes the HDR image into a detail and a coarse component by applying bilateral filtering. The watermark embedding is then performed in the detail component that preserves the images' edges. In fact, it is assumed that a generic TMO does not affect the image details and the color appearance, while addressing the problem of strong contrast reduction. Both methods proposed in [7] project the luminance of the detail component in the discrete wavelet domain. Then, the embedding is performed using spread-spectrum (SS) techniques. The experimental tests, conducted using five HDR images and four local TMOs, show that the visual quality of images marked with the $\mu$-law-based approach, evaluated by means of the peak signal-to-noise ratio (PSNR) computed over tone-mapped images, is better than the one associated with the method relying on bilateral filtering. Specifically, the PSNR of the images marked with the first approach is within the $50-70-\mathrm{dB}$ range, while the second one results in a PSNR of about 30$60 \mathrm{~dB}$. However, bilateral filtering guarantees better performance in terms of robustness to the application of TMOs. 
In [8], a general tone-mapping, represented by the logarithm function, is considered and applied to the luminance of the given HDR image, thus obtaining the LogLuv domain. The watermark embedding is performed by applying a quantization-index-modulation (QIM) approach [9] to the approximation subband of the discrete wavelet transform of the LogLuv component. Specifically, the image is divided into different blocks of random shapes, and the coefficients of each block are modified in order to make the block kurtosis equal to a non-uniformly quantized value determined by the bit to be embedded into the block. Mark imperceptibility is obtained by applying a local perceptual mask based on luminance, texture, and contrast that provides the maximum amount of distortion that each coefficient in the embedding domain can withstand without resulting in visible artifacts. Experimental tests conducted on 15 HDR images include an objective analysis on mark imperceptibility evaluated through the HDR-Visual Difference Predictor (HDR-VDP) metric [10] and on the robustness of watermark detection against seven different TMOs as well as to the addition of Gaussian noise in the HDR domain.

In [11], a non-linear hybrid embedding approach operating in the detail subbands of a one-level wavelet decomposition is used to watermark an HDR image. Specifically, the approach combines both additive and multiplicative watermark embedding and is based on a square-root embedding equation operating in the wavelet domain. The embedding approach is applied to 12 images, and its robustness is tested against the application of seven TMOs. The quality assessment is carried out using both the HDR-VDP metric and a pool of three observers on a native HDR display.

In [12], a bracketing approach mimicking the HDR image generation process, consisting of merging several single-exposure LDR images, is exploited for HDR image watermarking. In details, an HDR image is split into multiple LDR images, which are watermarked before being fused again into a single HDR image representation. The mark embedded in the HDR image thus generated results to be detectable in its LDR counterparts obtained by either applying a tone-mapping process or extracting and displaying only a specific range of interest of intensities. The HDR-VDP-2 metric [13] is employed to assess the performance. An equal error rate (EER) lower than $10^{-8} \%$ is achieved when detecting a watermark from LDR images obtained with five different TMOS. EERs lower than $10^{-2} \%$ are estimated when analyzing LDR images obtained by isolating, from the HDR data, a dynamic range different from the one considered during embedding.

A summary of the HDR image 1-bit watermarking approaches is given in in Table 1 .

\subsection{HDR image watermarking: multi-bit embedding}

In [14], binary information is inserted into an HDR image encoded in RGBe format by substituting the least significant bits (LSBs) of each pixel with bits taken from the secret message. In more detail, the HDR image is divided into flat and boundary areas by comparing the exponents associated to neighboring pixels. The number of bits which can be embedded into each color channel is then adaptively determined on the basis of local contrast and depending on the considered area, embedding more bits in high contrast and dark regions than in smooth and bright ones. The method capacity, tested on seven HDR images, is around 10 bits per pixel (bpp). Its imperceptibility is evaluated by converting the HDR images into their LDR counterparts by means of a TMO and measuring the peak signal-to-noise ratio (PSNR) between the tonemapped cover image and the tone-mapped watermarked image, obtaining values around $30 \mathrm{~dB}$.

Images in uncompressed LogLuv TIFF format are considered in [15]. The LSB of each channel's mantissa is modified to insert a binary message, while the exponent is selected in order to minimize the difference between the final value and the original one. The visual quality of the resulting images is evaluated over seven HDR images by applying tone-mapping and computing the PSNR and the HDR-VDP metrics.

The RGBe format is considered in [16], where its property of expressing a given color according to different possible choices is employed to hide a message into an HDR image: the final color representation is selected among the equivalent ones on the basis of the bits of the secret message. Such an approach produces distortionfree watermarked images, being different with the original cover image due only to the conversion employed to determine the floating point value of the pixels. A capacity of about $0.12 \mathrm{bpp}$ is estimated over five HDR images.

The blue component of a detail layer obtained exploiting bilateral filtering as in [7] is employed in [17] to embed into an HDR image binary messages with as many bits as the image's pixels. The mark is inserted additively yet proportionally to the pixels' luminance. The mark imperceptibility is measured only through the PSNR, evaluated over five HDR images, achieving values around $60 \mathrm{~dB}$. No objective measurements of mark extraction capability are provided, and the robustness against TMO is not evaluated. It is worth noting that no intentional or unintentional attack is taken into account in any of the aforementioned approaches performing multi-bit message embedding.

This kind of analysis is performed for the first time in [18]. The preprocessing stage proposed in [7], consisting of splitting an HDR image into its LDR counterpart and a residual component, is employed also here. Four TMOs are taken into account for both embedding the watermark 
Table 1 HDR image 1-bit watermarking: state of the art

\begin{tabular}{|c|c|c|c|c|c|}
\hline Paper & Embedding domain & Embedding method & \# of images database & Imperceptibility & Robusteness \\
\hline & & & & & 4TMOs \\
\hline \multirow[t]{3}{*}{ Xue et al. [7] } & $\mu$-law and wavelet & Multiplicative & $5^{a}$ & PSNR on LDR $\sim 55 \mathrm{~dB}$ & $\begin{array}{l}\text { Score }>\text { threshold at } \\
P_{f a}=10^{-8 \%}\end{array}$ \\
\hline & Bilateral and wavelet & Multiplicative & & PSNR on LDR $\sim 45 \mathrm{~dB}$ & $\begin{array}{l}\text { Score }>\text { threshold at } \\
P_{f a}=10^{-8 \%}\end{array}$ \\
\hline & & & & & $7 \mathrm{TMOs}$ \\
\hline \multirow[t]{3}{*}{ Guerrini et al. [8] } & LogLuv and wavelet & QIM & $15^{a, b}$ & $\mathrm{HDR} \mathrm{VDP}_{75} \sim 0.46 \%$ & $\begin{array}{l}P_{\text {miss }}=10^{-2} \% \text { at } \\
P_{f a}=10^{-6 \%}\end{array}$ \\
\hline & & & & $\mathrm{HDR}^{-V_{D P}} 95 \sim 0.21 \%$ & Add. noise \\
\hline & & & & & $\begin{array}{l}P_{\text {miss }}=10^{-12} \% \text { at } \\
P_{f a}=10^{-6 \%}\end{array}$ \\
\hline \multirow[t]{2}{*}{ Autrusseau et al. [11] } & Wavelet & Hybrid & $5,7^{a, b}$ & HDR-VDP & $8 \mathrm{TMOs}$ \\
\hline & & & & Subjective (3 subjects) & qualitative analysis \\
\hline Solachidis et al. [12] & Wavelet & Additive & $6^{\mathrm{a}}$ & HDR-VDP-295 $~ 5.00 \%$ & 6TMOs \\
\hline
\end{tabular}

ahttp://www.anyhere.com/gward/hdrenc/pages/originals.html

bhttp://www.cis.rit.edu/fairchild/HDRPS/HDRthumbs.html 
and attacking the marked HDR image when extracting the hidden data. Differently from [7], a multi-bit mark is blindly embedded into an image by associating each bit to a specific $8 \times 8$ luminance block and by manipulating two of its middle-frequency discrete cosine transform (DCT) coefficients to make their difference dependent on the bit to be embedded. Tests performed on four HDR images provide a PSNR for tone-mapped watermarked images of about $40 \mathrm{~dB}$, while a bit-error-rate (BER) of about $5 \%$ is estimated between the mark embedded in the HDR image and the one extracted from the tone-mapped watermarked image. Cropping, Gaussian blur, and Gaussian noise addition are considered as attacks to the LDR marked images, resulting in BERs of about 12, 23, and $15 \%$, respectively.

In [19], a blind multi-bit watermarking method for HDR images is proposed. The employed embedding domain is obtained by applying the wavelet transform to the justnoticeable difference (JND)-scaled representation of the original HDR image. Such domain is exploited in order to process values directly associated with specific characteristics of the HVS. A visual mask based on bilateral filtering is used for the embedding region selection while the watermark intensity in each wavelet decomposition subband is modulated according to a contrast sensitivity function. An extensive set of experimental tests is conducted considering three HDR images and seven TMOs. The imperceptibility in the HDR domain is evaluated through the HDR-VDP-2 metric, while an EER lower than $10^{-3 \%}$ with a BER around $5 \%$ is estimated over tonemapped LDR images.

A summary of the HDR image multi-bit watermarking approaches is given in Table 2.

\section{HDR image watermarking in the Radon DCT domain}

The multi-bit embedding method designed by the authors in [6] is further explored in the following, and a more extensive set of experimental results is here presented.

Let us consider a message $\mathbf{m}$ of $M$ bits to be embedded in an HDR image. With the goal to design a watermarking approach robust against non-malicious TMO application, we resort to perform mark embedding in the image edge region, since TMOs typically preserve the edges of the original image, whereas compressing its dynamic range and adapting it for rendering HDR images on devices with low contrast capabilities $[20,21]$. In order to achieve the desired goal, the properties of the Radon DCT (RDCT) domain are here exploited. As detailed in [22], the RDCT is a two-stage transform that consists in performing, as a first step, the Radon transform which maps each line of the host image into a single point. The DCT follows, therefore, the energy of the Radon projection concentrated in the first coefficients of the resulting RDCT representation, which can be eventually used as the host signal where to embed the watermark. The proposed watermarking scheme is given in Fig. 1 and details on the watermark embedding and extraction are given in the following sections.

\subsection{HDR embedding domain}

Without any loss of generality, we assume that the original HDR image is represented in the $R G B$ color space. The embedding is performed in the luminance component $\mathbf{Y}$. Actually, most of TMOs perform alterations in the luminance component $\mathbf{Y}$ while compressing the original dynamic range, often resulting in unpredictable alterations of the chrominance contributions. Therefore, the chrominance components are not suitable to host the watermark. More specifically, in our approach, we consider the LogLuv domain [8], derived from the application of the logarithm to the luminance $\mathbf{Y}$. The logarithmic operation simulates a specific property of the human visual system (HVS), that is, its higher sensitivity to contrast in shadow areas, whose dynamic is expanded by the logarithm, when compared to the contribution of highlights, which is on the contrary compressed. The resulting representation is then linearly normalized for generating an image $\mathbf{N}$ whose pixel values belong to the interval $[0,1]$.

A discrete wavelet transform (DWT) decomposition, mimicking the processing performed by the HVS [23], of the image $\mathbf{N}$ is then performed, and one of the resulting subbands $\mathbf{N}_{\gamma}$, with $\gamma \in\{L H, H L, H H\}$, is selected.

The considered subband is then decomposed into $B$ square blocks $\mathbf{f}^{(b)}$ with size $P \times P, b=1, \ldots, B$, in order to perform a local analysis of the image properties. A message $\mathbf{m}^{(b)}=\left[m_{1}^{(b)}, \ldots, m_{C}^{(b)}\right]$ derived taking $C$ bits from $\mathbf{m}$ is then embedded into each block $\mathbf{f}^{(b)}$ according to the processing illustrated in Fig. 2 and described in the following. Having indicated with $S_{1} \times S_{2}$ the size of the HDR image, the number of generated blocks is $B=\left\lfloor\frac{S_{1}}{2 P}\right\rfloor \cdot\left\lfloor\frac{S_{2}}{2 P}\right\rfloor$, while the length of the embedded message $\mathbf{m}$ is therefore equal to $M=B \cdot C$ bits.

The RDCT transform is applied to each block $\mathbf{f}^{(b)}$ extracted from $\mathbf{N}_{\gamma}$, with $b=1, \ldots, B$. The embedding of $C$ bits is then applied in the transformed domain as in [22]. Specifically, the employed implementation is based on the invertible and non-redundant finite Radon transform (FRAT) presented in [24]. Given a real-valued function $\mathbf{f}^{(b)}$ defined over a finite square grid $Z_{P}^{2}$, being $Z_{P}=$ $\{0,1, \cdots, P-1\}$, its FRAT $\mathbf{r}^{(b)}$ is defined as

$$
\mathbf{r}^{(b)}[k, l]=\frac{1}{\sqrt{P}} \sum_{(i, j) \in L_{k, l}} \mathbf{f}^{(b)}[i, j]
$$

where $L_{k, l}$ defines the set of points that forms a line on $Z_{P}^{2}$ : 
Table 2 HDR image multi-bit watermarking: state of the art

\begin{tabular}{|c|c|c|c|c|c|c|}
\hline Paper & Embedding domain & Embedding method & \# of images database & Capacity & Imperceptibility & Robusteness \\
\hline \multirow[t]{3}{*}{ Cheng et al. [14] } & RGBe & LSB & 7 & $\sim 10 \mathrm{bpp}$ & PSNR on LDR & N/A \\
\hline & & & N/A & & $(1 \mathrm{TMO}) \sim 30 \mathrm{~dB}$ & \\
\hline & & & & & PSNR on LDR & \\
\hline \multirow[t]{3}{*}{ Li et al. [15] } & LogLuv & LSB & 10 & $\sim 26 \mathrm{bpp}$ & $(1 \mathrm{TMO}) \sim 35 \mathrm{~dB}$ & N/A \\
\hline & & & N/A & & $\mathrm{HDR} \mathrm{VDP}_{75} \sim 0.42 \%$ & \\
\hline & & & & & HDR-VDP $95 \sim 0.04 \%$ & \\
\hline \multirow[t]{2}{*}{ Yu et al. [16] } & RGBe & Pixel & 125 & $\sim 0.12 \mathrm{bpp}$ & N/A & N/A \\
\hline & & exponent & N/A & & & \\
\hline \multirow[t]{2}{*}{$\begin{array}{l}\text { Rattanacharuchinda } \\
\text { et al. [17] }\end{array}$} & $\begin{array}{l}\text { Blue comp. of } \\
\text { detail layer }\end{array}$ & Multiplicative & $5^{\mathrm{a}}$ & $1 \mathrm{bpp}$ & PSNR 60 db & N/A \\
\hline & & & & & & $4 \mathrm{TMO}$ BER 5\% \\
\hline \multirow[t]{3}{*}{ Wu et al. [18] } & $\mathrm{DCT}$ & Modify & $4^{\mathrm{a}}$ & & PSNR on LDR & HDR crop BER 12\% \\
\hline & & adjacent & & $0.015 \mathrm{bpp}$ & (4 TMO) $[40,70] \mathrm{dB}$ & HDR blur BER $22 \%$ \\
\hline & & DCT coeff. & & & & HDR noise BER $15 \%$ \\
\hline Solachidis et al. [19] & $\begin{array}{l}\text { JND } \\
\text { wavelet }\end{array}$ & Multiplicative & $3^{b}$ & 128 & $\begin{array}{l}\text { HDR-VDP-2 } \sim 1 \% \\
(1 \mathrm{TMO}) \sim 30 \mathrm{~dB}\end{array}$ & $\begin{array}{l}7 \text { TMOs } \\
\text { EER }<10^{-3} \% \text { BER } \sim 5 \%\end{array}$ \\
\hline
\end{tabular}




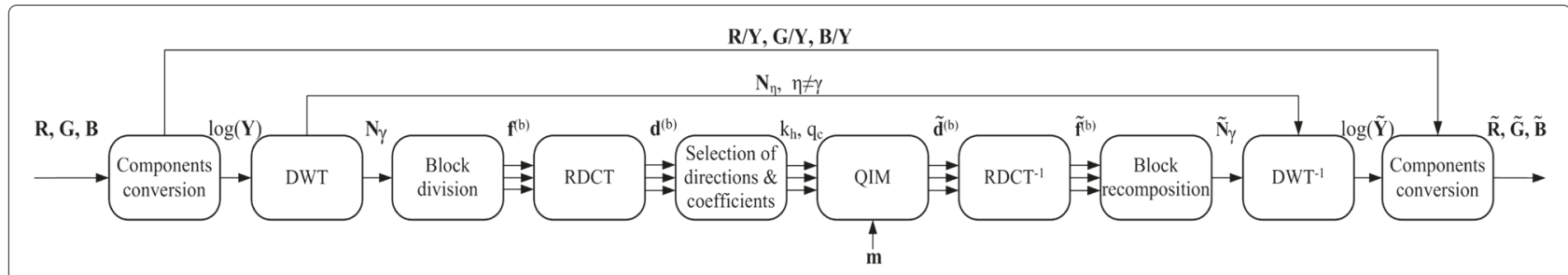

Fig. 1 Proposed embedding scheme

$$
\left\{\begin{array}{l}
L_{k, l}=\left\{(i, j): j=k \cdot i+l(\bmod P), i \in Z_{P}\right\} \\
L_{P, l}=\left\{(l, j): j \in Z_{P}\right\}
\end{array}\right.
$$

being $k \in Z_{P+1}$ the line direction and $l \in Z_{P}$ its intercept.

The Radon projections, obtained as in (1), are further processed in order to concentrate their energies in few coefficients. This goal is achieved by applying the DCT to the Radon projections, thus obtaining the RDCT sequences as

$$
\mathbf{d}^{(b)}[k, q]=\omega[l] \sum_{l=0}^{P-1} \mathbf{r}^{(b)}[k, l] \cos \left[\frac{\pi(2 l+1) q}{2 P}\right]
$$

with $q \in Z_{P}, \omega[0]=\sqrt{1 / P}$, and $\omega[l]=\sqrt{2 / P}, l \neq 0$. The energy concentration provided by the RDCT is similar to that performed by the ridgelet transform [24], yet with a better behavior and performance for watermarking purposes provided by the RDCT, as shown in [22].

\subsection{Host coefficients selection and QIM embedding}

The host coefficients where to embed the mark are selected as those related to the presence of edges. This task is accomplished by considering the RDCT projections of a given block $\mathbf{f}^{(b)}$ and by sorting the $P+1$ directions available in $\mathbf{d}^{(b)}$ according to their energies in decreasing order and finally selecting $H$ directions for mark embedding. Specifically, the directions from the $h_{\max }$-th to the $h_{\min }$-th most energetic ones, with $H=$ $h_{\min }-h_{\max }+1$, are selected to embed the $C$ bits of $\mathbf{m}^{(b)}$ in each of them.

Having indicated with $\mathbf{d}^{(b)}\left[k_{h}, q\right]$ the RDCT projection with the $h$-th most energetic contribution, with $h \in\left\{h_{\max }, \ldots, h_{\min }\right\}, C$ coefficients are taken from it and modified through QIM [9] to embed the desired message. It is worth specifying that, before doing this, the values in the considered RDCT projection are

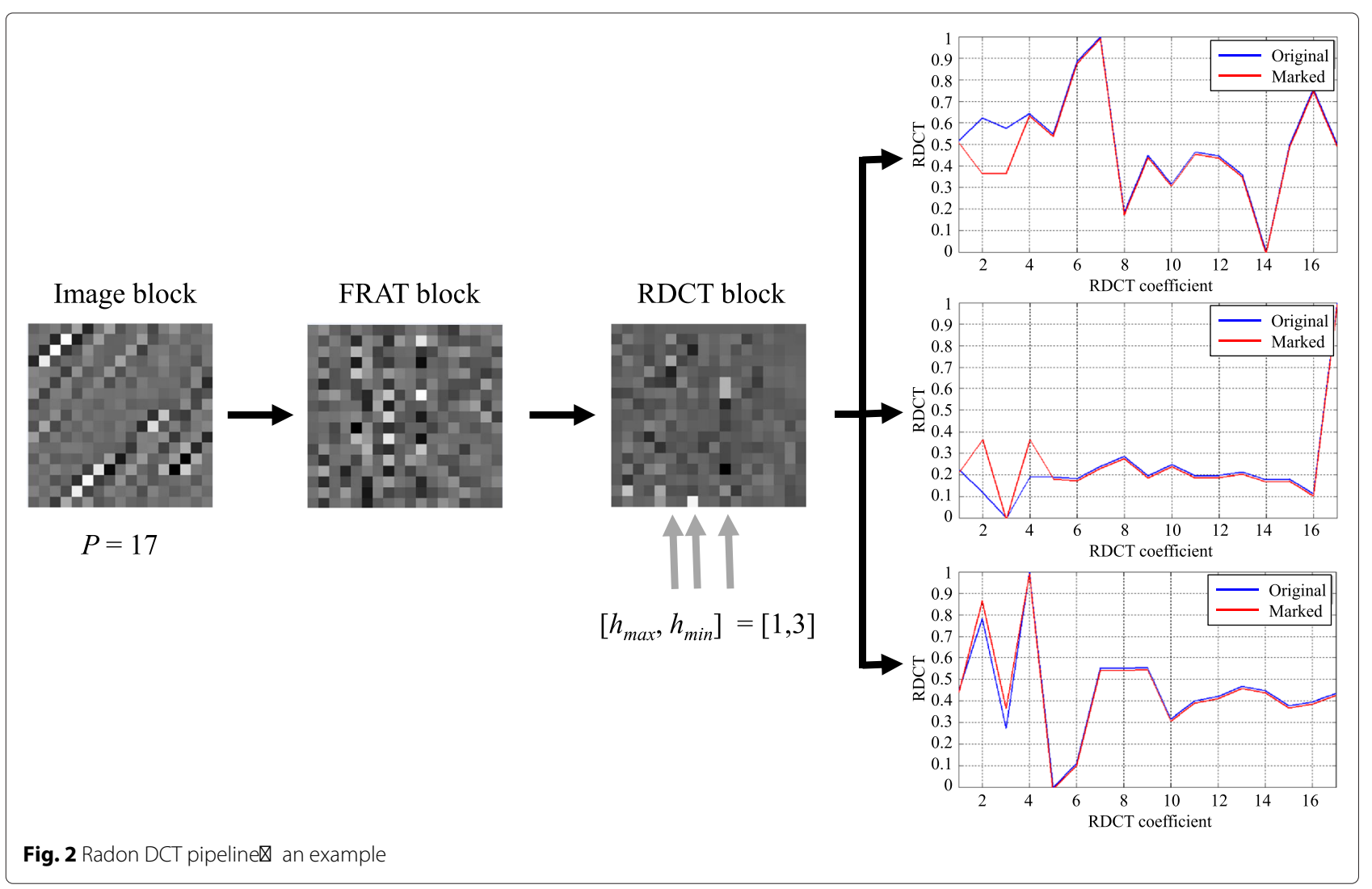


linearly normalized between 0 and 1 , thus producing the projections $\mathbf{d}_{N}^{(b)}\left[k_{h}, q\right]$. The coefficients having extreme values ( 0 and 1 after normalization) are not modified to not alter the range of the original values. Also, the RDCT coefficient $\mathbf{d}_{N}^{(b)}\left[k_{h}, 0\right]$, representing the DC component of the Radon projection, cannot be modified being connected with the mean value of all the projections computed from $\mathbf{f}^{(b)}$, as pointed in [22]. Therefore, the $C$ coefficients to be marked are selected as the first ones of the available RDCT sequence, with the exception of the aforementioned elements, namely the DC coefficient and the extreme values.

The $c$-th bit of $\mathbf{m}^{(b)}$, with $c \in\{1, \ldots, C\}$, is therefore considered to alter the original coefficient $\mathbf{d}_{N}^{(b)}\left[k_{h}, q_{c}\right]$ according to a QIM approach (see Fig. 3). Specifically, keeping in mind that the considered coefficients lie in the interval $[0,1]$, two code books $\mathcal{U}_{0}$ and $\mathcal{U}_{1}$ containing quantized values associated, respectively, to $m_{c}^{(b)}=0$ and $m_{c}^{(b)}=1$ can be defined as

$$
\left\{\begin{array}{l}
\mathcal{U}_{0}=\left\{\frac{1+4 z}{2^{\Delta+1}}, z \in\left\{0, \ldots, 2^{\Delta-1}-1\right\}\right. \\
\mathcal{U}_{1}=\left\{\frac{3+4 z}{2^{\Delta+1}}, z \in\left\{0, \ldots, 2^{\Delta-1}-1\right\}\right.
\end{array}\right\}
$$

being $\Delta$ the natural integer determining the quantization step $\frac{1}{2^{\Delta}}$ between admissible quantized values.

Watermark embedding is achieved by applying to $\mathbf{d}_{N}^{(b)}\left[k_{h}, q_{c}\right]$ either the quantizer associated to $\mathcal{U}_{0}$ or the one associated to $\mathcal{U}_{1}$ :

$$
\left\{\begin{array}{l}
\mathcal{Q}_{0}\left(\mathbf{d}_{N}^{(b)}\left[k_{h}, q_{c}\right]\right)=\arg \min _{u_{0, z} \in \mathcal{U}_{0}}\left|u_{0, z}-\mathbf{d}_{N}^{(b)}\left[k_{h}, q_{c}\right]\right| \\
\mathcal{Q}_{1}\left(\mathbf{d}_{N}^{(b)}\left[k_{h}, q_{c}\right]\right)=\arg \min _{u_{1, z} \in \mathcal{U}_{1}}\left|u_{1, z}-\mathbf{d}_{N}^{(b)}\left[k_{h}, q_{c}\right]\right|
\end{array}\right.
$$

where $u_{0, z}$ and $u_{1, z}$ are elements of $\mathcal{U}_{0}$ and $\mathcal{U}_{1}$, respectively. The marked coefficient $\tilde{\mathbf{d}}_{N}^{(b)}\left[k_{h}, q_{c}\right]$ can be expressed as

$$
\tilde{\mathbf{d}}_{N}^{(b)}\left[k_{h}, q_{c}\right]= \begin{cases}\mathcal{Q}_{0}\left(\mathbf{d}_{N}^{(b)}\left[k_{h}, q_{c}\right]\right), & \text { if } m_{c}^{(b)}=0, \\ \mathcal{Q}_{1}\left(\mathbf{d}_{N}^{(b)}\left[k_{h}, q_{c}\right]\right), & \text { if } m_{c}^{(b)}=1 .\end{cases}
$$

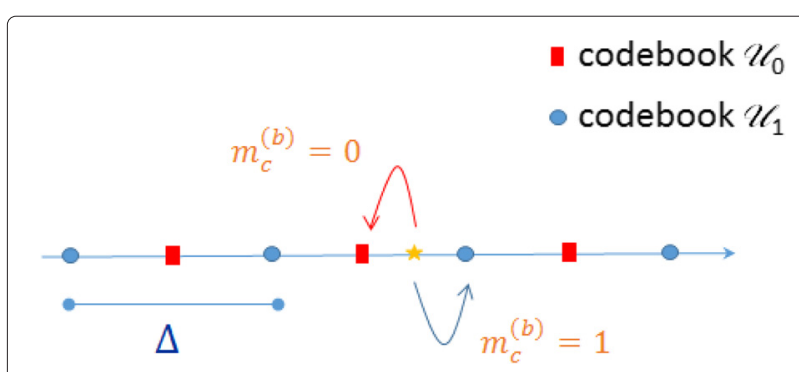

Fig. 3 QIM embedding procedure
The effects on the achievable performance, in terms of mark imperceptibility and robustness, of the employed parameters, namely the choice of the wavelet subband $\gamma$, the block size $P$, the embedding directions and coefficients, and the quantization parameter $\Delta$, are discussed in Section 5. As for the capacity achievable with the proposed approach, it is easy to express it in a closed form as $\frac{C}{4 \cdot P^{2}}$ bpp. Practical values of the lengths of messages which can be embedded into HDR images are given in Section 5.

\subsection{HDR image reconstruction}

As described in the previous section, the block $\tilde{\mathbf{d}}_{N}^{(b)}$ is obtained from $\mathbf{d}_{N}^{(b)}$ by marking $C$ coefficients of each direction $k_{h}$, with $h \in\left\{h_{\max }, \ldots, h_{\min }\right\}$. The RDCT representation $\tilde{\mathbf{d}}^{(b)}$ is derived by reversing the normalization process performed during the embedding. The watermarked block $\tilde{\mathbf{f}}^{(b)}$ is then determined through an inverse RDCT. The marked wavelet subband $\tilde{\mathbf{N}}_{\gamma}$ is reconstructed by recombining all the $B$-marked blocks. The marked normalized luminance $\tilde{\mathbf{N}}$ is then generated by combining $\tilde{\mathbf{N}}_{\gamma}$ with the other unaltered subbands $\tilde{\mathbf{N}}_{\eta}, \eta \neq \gamma$, and performing the inverse wavelet transform. The normalization process done during the embedding is reversed by applying an exponential operator thus obtaining the marked luminance $\tilde{\mathbf{Y}}$ which, as a result, is characterized by the same dynamic range of the original luminance $\mathbf{Y}$. The final watermarked image is generated by defining its trichromatic components as $\tilde{\mathbf{R}}=\frac{\mathbf{R}}{\mathbf{Y}} \cdot \tilde{\mathbf{Y}}, \tilde{\mathbf{G}}=\frac{\mathbf{G}}{\mathbf{Y}} \cdot \tilde{\mathbf{Y}}$ and $\tilde{\mathbf{B}}=\frac{\mathbf{B}}{\mathbf{Y}}$. $\tilde{\mathbf{Y}}$. Therefore, the original chrominance contributions are kept unaltered, while the luminance of the resulting image allows to blindly recover the embedded information.

\subsection{Mark extraction}

In the watermark extraction stage, the marked HDR image undergoes the same operations described in Section 4.1 thus obtaining the RDCT projections $\overline{\mathbf{d}}^{(b)}\left[k_{h}, q\right]$ with $h \in\left\{h_{\max }, \ldots, h_{\min }\right\}$. Each projection is first normalized between 0 and 1 , thus obtaining $\overline{\mathbf{d}}_{N}^{(b)}$, and then, the embedded bit estimation $\hat{m}_{h, c}^{(b)}, c=1, \ldots, C$ is performed using a minimum distance decoder:

$$
\begin{array}{r}
\hat{m}_{h, c}^{(b)}=\arg \min _{x \in\{0,1\}} \min _{u_{x, z} \in \mathcal{U}_{x}}\left|u_{x, z}-\overline{\mathbf{d}}_{N}^{(b)}\left[k_{h}, q_{c}\right]\right|, \\
z \in\left\{0, \ldots, 2^{\Delta-1}-1\right\} .
\end{array}
$$

The final estimations $\hat{m}_{c}^{(b)}, c=1, \ldots, C$, of the $C$ bits embedded into the block $\overline{\mathbf{d}}^{(b)}$ are made according to a majority-voting decision evaluated over the $H$ estimates $\hat{m}_{h, c}^{(b)}, h \in\left\{h_{\max }, \ldots, h_{\min }\right\}$, available for each embedded bit.

It is worth specifying that the proposed approach takes into account more than a single direction for embedding $C$ bits in each block to properly manage errors during the extraction process. Specifically, a relevant source of errors 
comes from the possibility of selecting for a block, during the mark extraction process, projections which differ from those employed during the embedding. In fact, if the energies of the RDCT projections of a given block are modified by some processing applied to the marked HDR image, the order with which the projections are selected to extract the mark may be altered, making it hard to recover the bits inserted into the considered block. The employed majority-voting decision strategy allows mitigating the effects of such events.

The considered approach allows also retrieving the embedded information from a tone-mapped LDR version of the watermarked HDR image. However, in this scenario, the mark is extracted directly from the luminance of the considered LDR image and not from its logarithmic counterpart. As in [8], it is in fact assumed that the application of a TMO produces a perceptual processing similar to the one performed by the logarithm operator.

\section{Experimental results}

An extensive set of experimental tests has been carried out to evaluate the performance of the proposed blind watermarking approach for HDR images. Specifically, to this aim, we have exploited 15 HDR images collected from http://www.mpi-inf.mpg.de/resources/hdr/ gallery.html and http://www.anyhere.com/gward/hdrenc/ pages/originals.html. The main characteristics of the employed images are listed in Table 3, while Fig. 4 shows all of them through their LDR representations, obtained with the ICAM06 TMO [25]. As can be seen, in the performed tests, we have considered images with different dimensions, dynamic ranges, and amount of edges and textured areas, in order to investigate the performance of the proposed method under a significant variability of possible practical conditions. Moreover, all the employed images have been selected to represent the depicted scenarios through real luminance values expressed in $\mathrm{cd} / \mathrm{m}^{2}$, being thus possible to exploit the HDR-VDP-2 metric [13] to objectively evaluate the imperceptibility of the embedded watermarks. In more details, the HDR-VDP-2 metric provides, for each pixel of a modified image, the probability of being detected as different from the original representation, that is, the percentage of people recognizing the pixel being modified. The latest HDR-VDP-2 version currently available (ver. 2.2.1) is employed in the performed tests.

The embedding capacities practically achievable for the considered images are shown in Table 4, for different values of $P$ and using $C=3$. As can be seen, the size of the embedded messages can grow very large, depending on the dimensions of the selected HDR image.

Table 4 also reports the processing time required for embedding messages into an HDR image according to the
Table 3 Employed HDR images

\begin{tabular}{llll}
\hline $\begin{array}{l}\text { Image } \\
\text { ID }\end{array}$ & $\begin{array}{l}\text { Image } \\
\text { name }\end{array}$ & $\begin{array}{l}\text { Dynamic } \\
\text { range }\end{array}$ & $\begin{array}{l}\text { Size } \\
\left(S_{1} \times S_{2}\right)\end{array}$ \\
\hline 1 & AtriumMorning & $1.99 \cdot 2^{14}$ & $1016 \times 760$ \\
2 & AtriumNight & $1.62 \cdot 2^{28}$ & $1016 \times 760$ \\
3 & mpi_atrium_1 & $1.48 \cdot 2^{14}$ & $676 \times 1024$ \\
4 & nancy_cathedral_1 & $1.60 \cdot 2^{14}$ & $2048 \times 1536$ \\
5 & nancy_cathedral_2 & $1.94 \cdot 2^{14}$ & $2048 \times 1536$ \\
6 & nave & $1.64 \cdot 2^{23}$ & $480 \times 720$ \\
7 & archway & $1.75 \cdot 2^{7}$ & $1080 \times 721$ \\
8 & medway & $1.99 \cdot 2^{8}$ & $1080 \times 723$ \\
9 & kitchenwindow & $1.77 \cdot 2^{11}$ & $1080 \times 1650$ \\
10 & memorial & $1.29 \cdot 2^{18}$ & $768 \times 512$ \\
11 & rosette & $1.83 \cdot 2^{17}$ & $480 \times 720$ \\
12 & snow & $1.02 \cdot 2^{10}$ & $1536 \times 2048$ \\
13 & rend07 & $1.41 \cdot 2^{28}$ & $575 \times 575$ \\
14 & rend10 & $1.20 \cdot 2^{26}$ & $1024 \times 1024$ \\
15 & Iwate & $1.08 \cdot 2^{19}$ & $1396 \times 3720$ \\
\hline
\end{tabular}

proposed method, when using Matlab [26] on a computer equipped with an Intel(R) Core(TM) i7-4500U at $1.8 \mathrm{GHz}$ and 8.0 GB RAM. Given an HDR image, the required processing time is mainly dependent on the adopted block size $P$, and only the dependency on this parameter is therefore shown in Table 4 , which provides values obtained with $\Delta=2,\left[h_{\max }, h_{\min }\right]=[1,5]$, and $C=3$. As can be seen, large values of $P$ are preferable for speeding up the marking process.

An analysis of the imperceptibility performance guaranteed by the proposed approach is then provided in Table 5 , where the dependency on the employed parameters is investigated. Specifically, the table reports the maximum probability of detecting differences between the original and the marked data in at least the $5 \%$ of the image pixels, for different choices of $P, \Delta,\left[h_{\max }, h_{\min }\right]$ and $C$. It is worth specifying that the reported results are always referred to the mark embedding in the $\gamma=H H$ subband, since it provides far better imperceptibility performance with respect to the other first-level DWT subbands $L H$ and $H L$. Actually, the evaluated probability of detecting a modification in at least $5 \%$ of the image is $1.96 \%$ when $\gamma=H H$, averaged over all the considered selections for the directions to be marked and the number of modified coefficients, with $P=17$ and $\Delta=2$. On the other hand, the same probability is $41.35 \%$ when using the horizontal detail subband $\gamma=H L$ and reaches $53.13 \%$ for the vertical detail subband $\gamma=L H$.

As can be seen from the results reported in Table 5, although decreasing the block dimension $P$ would imply the possibility of embedding more bits in a given HDR 


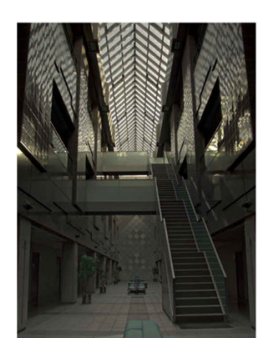

1

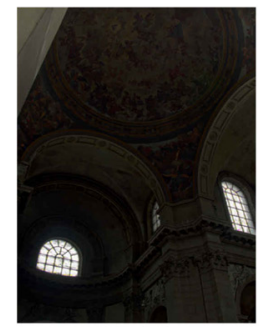

4

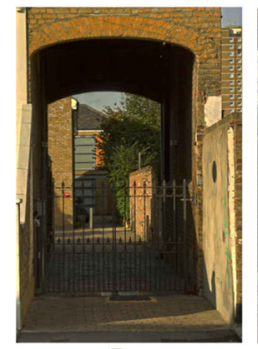

7

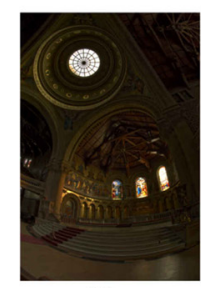

10

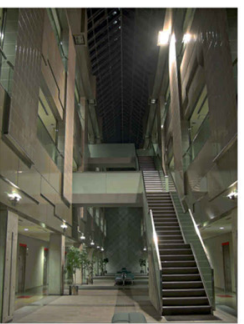

2

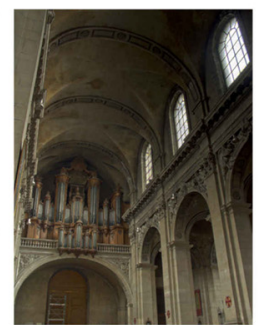

5

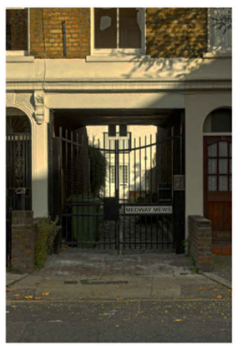

8

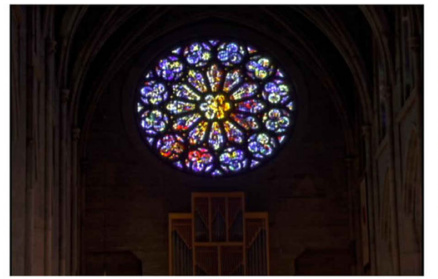

11

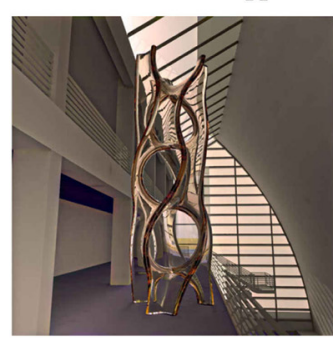

13

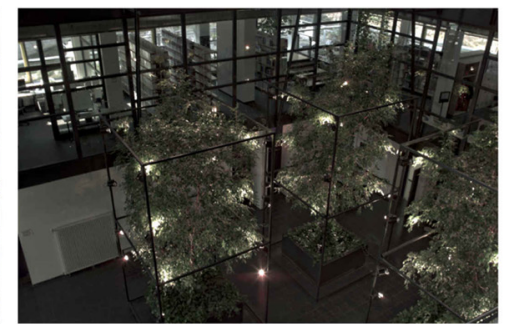

3

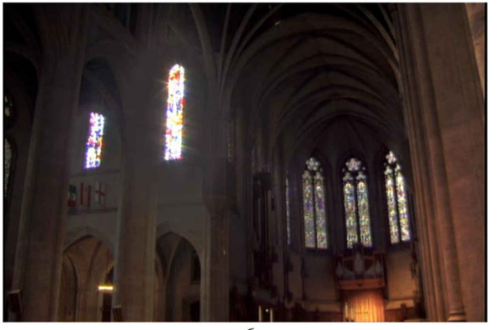

6

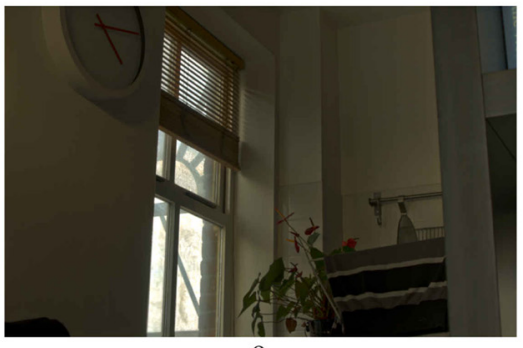

9

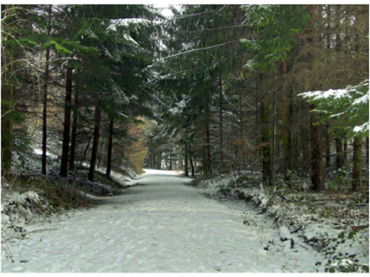

12

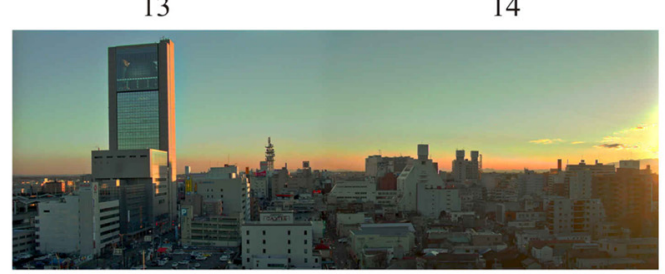

15

Fig. 4 Employed HDR images, rendered through the ICAM06 TMO [25] 
Table 4 Capacity, in bpp and embeddable bits, of the proposed method, for $C=3$. Also, the required embedding processing time is provided

\begin{tabular}{|c|c|c|c|c|c|c|c|c|c|c|c|c|c|c|c|c|}
\hline & \multirow{2}{*}{$P$} & \multicolumn{15}{|c|}{ Image } \\
\hline & & 1 & 2 & 3 & 4 & 5 & 6 & 7 & 8 & 9 & 10 & 11 & 12 & 13 & 14 & 15 \\
\hline Capacity & 7 & & & & & & & & 0.0153 & & & & & & & \\
\hline \multirow[t]{2}{*}{ (bpp) } & 11 & & & & & & & & 0.0062 & & & & & & & \\
\hline & 17 & & & & & & & & 0.0026 & & & & & & & \\
\hline Capacity & 7 & 11664 & 11664 & 10512 & 47742 & 47742 & 5202 & 11781 & 11781 & 27027 & 5832 & 5202 & 47742 & 5043 & 15987 & 78705 \\
\hline \multirow[t]{2}{*}{$(M)$} & 11 & 4692 & 4692 & 4140 & 19251 & 19251 & 2016 & 4704 & 4704 & 11025 & 2346 & 2016 & 19251 & 2028 & 6348 & 31941 \\
\hline & 17 & 1914 & 1914 & 1710 & 8100 & 8100 & 882 & 1953 & 1953 & 2346 & 990 & 882 & 8100 & 768 & 2700 & 13407 \\
\hline Processing & 7 & 33.8 & 33.8 & 29.9 & 130.3 & 130.3 & 12.7 & 33.9 & 33.9 & 73.3 & 16.7 & 12.7 & 130.3 & 12.5 & 41.4 & 220.3 \\
\hline \multirow[t]{2}{*}{ time (s) } & 11 & 14.5 & 14.5 & 12.6 & 57.4 & 57.4 & 5.3 & 14.6 & 14.6 & 32.9 & 7.2 & 5.3 & 57.4 & 5.2 & 18.1 & 97.4 \\
\hline & 17 & 6.6 & 6.6 & 5.7 & 26.9 & 26.9 & 2.6 & 6.6 & 6.6 & 14.7 & 3.3 & 2.6 & 26.9 & 2.4 & 8.7 & 45.1 \\
\hline
\end{tabular}

image as shown in Table 4, it would also result in an increased probability of detecting a modification in the marked HDR image. The obtained results also show that the embedded mark is more imperceptible as the parameter $\Delta$ is set to higher values, since a smaller quantization step $\frac{1}{2^{\Delta}}$ is thus obtained. Moreover, selecting the $H=$ 3 first most energetic directions to embed the mark in each image block guarantees a lower perceptual impact than using $H=5$ directions. Within this regard, it can be also noticed that mark imperceptibility can be improved by discarding the most energetic projection for the embedding, as it is shown by the results achieved with $\left[h_{\max }, h_{\min }\right]=[2,4]$ and $\left[h_{\max }, h_{\min }\right]=[2,6]$. Eventually, the increase of the number of the marked coefficients $C$ increases the number of embedded bits at the cost of worsening the imperceptibility performance.

A visual example on the imperceptibility of the embedded mark is given in Fig. 5, where the original image 1 is shown together with its processed version with $\gamma=H H$, $P=17,\left[h_{\max }, h_{\min }\right]=[1,5], C=3$ and $\Delta=2$. The reported LDR representations are obtained by means of the ICAM06 TMO [25]. From Table 5, it can be seen that such choice of system parameters allows to guarantee, on average, a probability of detecting a modification in at least $5 \%$ of the image which is equal to $6.4 \%$. In order to further demonstrate the effectiveness of the proposed approach, the achieved performance is compared with the one provided by the method in [18], the only one so far proposed that allows to embed large messages (>1000 bits) in an HDR image, while being able to recover them from tone-mapped marked images, as reported in Table 2. It has been noticed that, when applying the method in [18] to the 15 images considered for our experiments, the average probability of detecting a modification in at least $5 \%$ of the marked images, measured with the HDR-VDP-2 metric, is equal to $27.6 \%$, being therefore

Table 5 Imperceptibility performance expressed through the HDR-VDP-2 metric (in \%). Results averaged over the considered images

\begin{tabular}{|c|c|c|c|c|c|c|c|}
\hline \multirow[b]{2}{*}[h_{\operatorname{max}},h_{\operatorname{min}}]{} & \multirow[b]{2}{*}{$C$} & \multicolumn{2}{|c|}{$P=7$} & \multicolumn{2}{|c|}{$P=11$} & \multicolumn{2}{|c|}{$P=17$} \\
\hline & & $\Delta=1$ & $\Delta=2$ & $\Delta=1$ & $\Delta=2$ & $\Delta=1$ & $\Delta=2$ \\
\hline \multirow[t]{3}{*}[1,3]{} & 1 & 16.38 & 2.51 & 11.77 & 1.32 & 6.04 & 0.44 \\
\hline & 2 & 28.18 & 5.79 & 16.32 & 3.23 & 9.96 & 1.21 \\
\hline & 3 & 36.49 & 8.42 & 30.06 & 5.50 & 18.12 & 2.33 \\
\hline \multirow[t]{3}{*}[2,4]{} & 1 & 15.69 & 1.48 & 7.51 & 0.87 & 6.20 & 0.50 \\
\hline & 2 & 23.59 & 3.27 & 11.79 & 1.59 & 9.53 & 1.31 \\
\hline & 3 & 29.51 & 8.41 & 24.57 & 4.48 & 15.44 & 2.10 \\
\hline \multirow[t]{3}{*}[1,5]{} & 1 & 20.30 & 3.78 & 14.80 & 2.43 & 7.55 & 1.03 \\
\hline & 2 & 35.00 & 8.55 & 26.85 & 4.54 & 16.51 & 1.79 \\
\hline & 3 & 48.79 & 16.13 & 33.59 & 9.42 & 19.17 & 6.40 \\
\hline \multirow[t]{3}{*}[2,6]{} & 1 & 16.92 & 1.70 & 13.30 & 1.78 & 8.09 & 0.69 \\
\hline & 2 & 31.48 & 6.97 & 16.29 & 4.59 & 12.08 & 2.35 \\
\hline & 3 & 36.68 & 15.19 & 26.50 & 8.38 & 18.82 & 3.40 \\
\hline
\end{tabular}




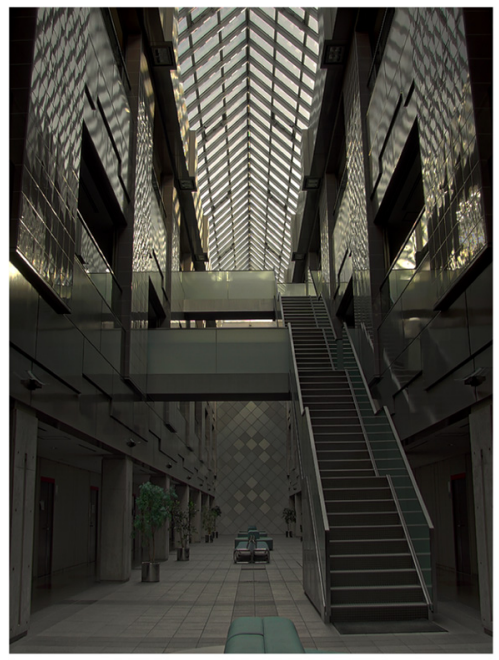

a

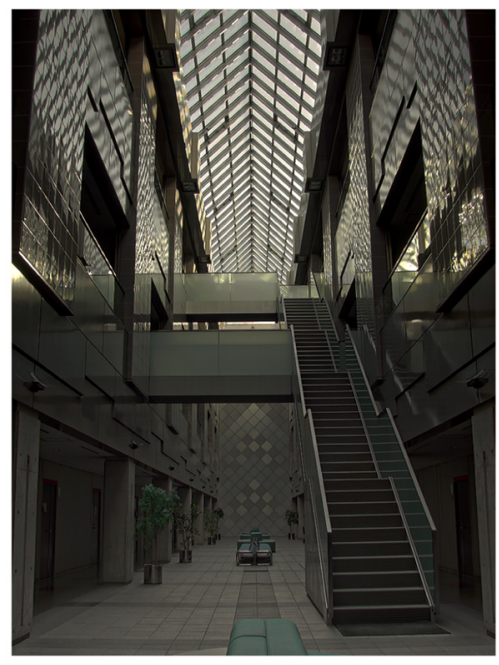

b

Fig. 5 Comparison between tone-mapped version of a: original image 1; b: marked image 1 (AtriumMorning)

much larger than the one obtained by the approach we propose. It is worth specifying that the parameters of the work in [18] have been selected in order to guarantee as much imperceptibility as possible, while still achieving admissible performance regarding data extraction from marked HDR images. The method in [18] in fact relies on the modification of a DCT coefficient in each $8 \times 8$ block of an LDR version of the considered HDR content. Nonetheless, large alterations are needed to achieve low BER values. The Durand TMO [20] is employed to generate the LDR images where the mark is embedded, as described in [18].

The parameter configuration with $\gamma=H H, P=$ 17, $\left[h_{\max }, h_{\min }\right]=[1,5], C=3$, and $\Delta=2$ is taken into account to also evaluate the robustness of the proposed approach. Within this regard, Table 6 reports the BERs obtained when retrieving the embedded marks from the marked HDR images, as well as from their LDR counterparts obtained with the six TMOs listed in the table. From the reported results, it is evident that some errors may be encountered even when analyzing the marked HDR images, mainly due to the modification of the directions energies when performing the embedding and to the conversions performed when saving the marked images into an HDR format. Moreover, it can be seen that the application of distinct TMOs may represent a very different attack for the extraction of the mark from LDR representations. For instance, with the selected configuration, the best performance is achieved when considering the Reinhard TMO [27], while significantly worse results are obtained with the tonemap operator provided in Matlab [26]. It can be argued that the Reinhard TMO provides the best performance since it is based on bilateral filtering, therefore trying to accurately maintain the edges of an HDR image when converting it into its LDR version. Being the proposed approach based on data embedding close to the edges, a TMO preserving these components is also able to preserve the information there hidden.

The comparison between the robustness performance of the proposed approach, when still maintaining the aforementioned system parameters, and the method in [18], is given in Table 7. It can be seen that the method in [18] performs quite similarly for all the considered TMOs, yet it can never provide results as good as those achieved with our approach. Mark extraction is hard from HDR images, too, when an admissible imperceptibility has to be guaranteed with the method in [18].

A more detailed analysis on the capability of extracting a mark from tone-mapped versions of the marked HDR images is obtained by evaluating the BER achievable for different system configurations. As for the imperceptibility analysis, Table 8 reports the performance obtained for different choices of $P, \Delta,\left[h_{\max }, h_{\min }\right]$, and $C$, while embedding the marks in the $\gamma=H H$ first-level decomposition subband.

Imperceptibility and robustness of the embedded marks are often conflicting requirements. This is experimentally confirmed by observing that the embedded marks can be extracted with less errors from LDR images obtained when using a lower value of $\Delta$, since the resulting quantization step is much larger, even if the imperceptibility is negatively affected. It can be also seen that selecting a large number of directions for embedding purposes improves 
Table 6 BER performance (in \%) for data retrieval from marked HDR and LDR images, when considering $\gamma=H H, P=17, \Delta=2$, $\left[h_{\max }, h_{\min }\right]=[1,5]$, and $C=3$

\begin{tabular}{|c|c|c|c|c|c|c|c|}
\hline \multirow[b]{2}{*}{ Image } & \multirow{3}{*}{ HDR } & \multicolumn{4}{|c|}{ TMO } & \multirow{3}{*}{$\begin{array}{c}\text { Tumblin } \\
\text { [29] }\end{array}$} & \multirow{3}{*}{$\begin{array}{c}\text { Durano } \\
\text { [20] }\end{array}$} \\
\hline & & iCAM06 & tonemap & Reinhard & Drago & & \\
\hline ID & & [25] & [26] & [27] & [28] & & \\
\hline 1 & 0.16 & 14.84 & 14.00 & 7.21 & 6.95 & 23.98 & 23.77 \\
\hline 2 & 0.57 & 9.87 & 21.00 & 4.18 & 3.45 & 9.56 & 10.03 \\
\hline 3 & 1.23 & 12.98 & 16.61 & 4.56 & 4.27 & 14.68 & 19.71 \\
\hline 4 & 6.69 & 31.26 & 27.65 & 18.68 & 25.26 & 19.38 & 15.33 \\
\hline 5 & 12.11 & 23.42 & 31.07 & 20.42 & 26.11 & 23.02 & 23.86 \\
\hline 6 & 29.12 & 36.26 & 36.26 & 33.08 & 33.88 & 33.99 & 36.58 \\
\hline 7 & 4.86 & 16.79 & 24.99 & 9.68 & 30.21 & 18.38 & 28.57 \\
\hline 8 & 1.64 & 13.36 & 22.38 & 8.14 & 17.05 & 15.92 & 19.41 \\
\hline 9 & 23.19 & 35.51 & 42.76 & 31.54 & 36.63 & 36.47 & 31.92 \\
\hline 10 & 0.03 & 15.15 & 15.66 & 5.86 & 8.48 & 8.28 & 7.17 \\
\hline 11 & 19.59 & 36.26 & 28.21 & 30.36 & 28.89 & 32.29 & 32.06 \\
\hline 12 & 0.17 & 5.42 & 17.89 & 6.96 & 10.38 & 14.79 & 19.23 \\
\hline 13 & 22.26 & 33.98 & 42.32 & 28.39 & 30.73 & 30.08 & 25.91 \\
\hline 14 & 22.22 & 32.81 & 33.52 & 24.19 & 30.44 & 30.22 & 27.70 \\
\hline 15 & 0.16 & 5.91 & 37.98 & 4.97 & 35.46 & 6.86 & 21.17 \\
\hline
\end{tabular}

the mark extraction capabilities, although slightly affecting the imperceptibility of the performed modifications. Moreover, excluding the most energetic direction during the embedding worsens the achievable BERs, even if it is beneficial from the imperceptibility point of view, for both $H=3$ and $H=5$. As for the number of coefficients $C$ marked for each direction, no meaningful difference in terms of BER can be noticed, thus suggesting to use $C=3$, even if at the cost of a higher visibility of the modifications. It has also to be noticed that, although increasing the block dimension $P$ significantly improves mark imperceptibility, it does not affect negatively the mark extraction capability, making thus preferable the selection of large block values such as $P=17$. With such a selection, and exploiting a system configuration with $\Delta=2,\left[h_{\max }, h_{\min }\right]=$ $[1,5]$, and $C=3$, it is possible to guarantee a probability of detecting a modification in at least the $5 \%$ of the image of about $6 \%$, while simultaneously achieving a BER for the mark extracted from LDR images at about $19 \%$, with marks in the order of at least a thousand of bits for the most of considerable images, as shown in Table 4.

It is also worth mentioning that even the selection of the DWT subband to be marked affects the robustness of the marking procedure: considering $P=17$ and $\Delta=2$, the BER obtained for $\gamma=H H$ is equal to $25.66 \%$, averaged over all the considered possibilities for marked directions and number of modified coefficients, while a better behavior is obtained when using the horizontal details $(\gamma=H L)$ subband achieving a BER $=15.31 \%$ and the vertical detail $(\gamma=L H)$ subband achieving a BER $=14.56 \%$. The trade-off between imperceptibility and robustness is confirmed by this analysis too.

Eventually, besides analyzing the robustness of the proposed approach against non-malicious attacks such as those performed by means of TMOs, we have also evaluated the behavior of our watermarking schemes against malicious signal processing attacks carried out on the marked HDR images, still considering a system

Table 7 Comparison between our method and the one in [18] for the BER performance (in \%) regarding data retrieval from marked HDR and LDR images, averaged over all the 15 considered images

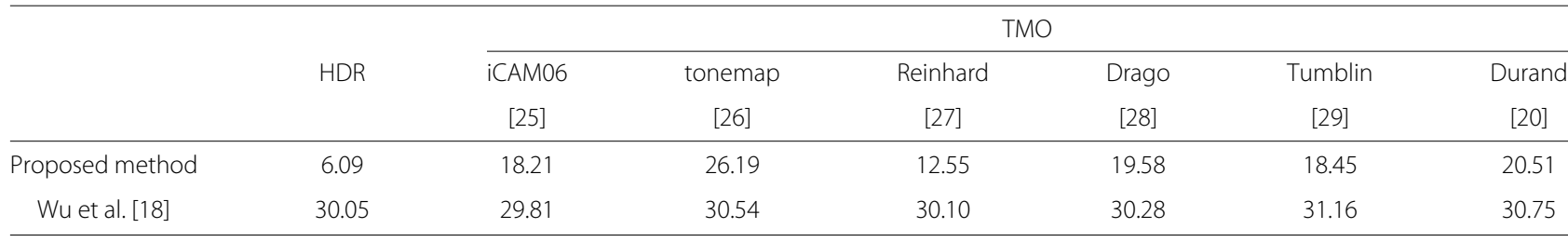


Table 8 Robustness performance in terms of BER (in \%). Results averaged over the considered images and TMOs

\begin{tabular}{|c|c|c|c|c|c|c|c|}
\hline \multirow[b]{2}{*}[h_{\operatorname{max}},h_{\operatorname{min}}]{} & \multirow[b]{2}{*}{$C$} & \multicolumn{2}{|c|}{$P=7$} & \multicolumn{2}{|c|}{$P=11$} & \multicolumn{2}{|c|}{$P=17$} \\
\hline & & $\Delta=1$ & $\Delta=2$ & $\Delta=1$ & $\Delta=2$ & $\Delta=1$ & $\Delta=2$ \\
\hline \multirow[t]{3}{*}[1,3]{} & 1 & 17.78 & 27.67 & 15.62 & 24.78 & 16.34 & 23.37 \\
\hline & 2 & 17.41 & 27.69 & 15.12 & 24.19 & 13.96 & 22.11 \\
\hline & 3 & 17.59 & 28.07 & 14.90 & 24.07 & 13.64 & 21.99 \\
\hline \multirow[t]{3}{*}[2,4]{} & 1 & 21.94 & 32.08 & 20.25 & 28.57 & 19.69 & 27.05 \\
\hline & 2 & 22.68 & 31.76 & 20.35 & 29.03 & 18.53 & 26.34 \\
\hline & 3 & 23.31 & 32.30 & 20.92 & 28.62 & 18.94 & 26.47 \\
\hline \multirow[t]{3}{*}[1,5]{} & 1 & 15.82 & 26.28 & 13.73 & 22.58 & 13.21 & 20.36 \\
\hline & 2 & 16.00 & 26.74 & 12.86 & 22.18 & 11.01 & 19.02 \\
\hline & 3 & 16.25 & 26.91 & 13.23 & 22.16 & 11.29 & 19.52 \\
\hline \multirow[t]{3}{*}[2,6]{} & 1 & 17.69 & 29.21 & 14.45 & 24.06 & 13.24 & 21.39 \\
\hline & 2 & 18.22 & 29.11 & 14.74 & 23.96 & 12.57 & 20.65 \\
\hline & 3 & 18.67 & 29.47 & 15.15 & 24.36 & 12.58 & 20.82 \\
\hline
\end{tabular}

configuration with $P=17, \Delta=2,\left[h_{\max }, h_{\min }\right]=[1,5]$, and $C=3$. Specifically, Table 9 presents the BER performance, averaged over all the considered 15 images, achieved when extracting the embedded messages from HDR images attacked with additive Gaussian noise and with Gaussian blurring. In more detail, Gaussian noise with a standard deviation equal to 3 has been added to the employed images, while the employed Gaussian blurring filter has been defined with a $[3,3]$ window and a standard deviation set to 0.3 . We have also investigated the possibility of extracting the embedded information from tone-mapped versions of the attacked HDR images. As the reported results testify, the proposed approach is able to provide proper resilience even against malicious signal processing attacks. Our method performs significantly better than the one in [18] when considering Gaussian filtering as an attack, while similar behaviors have been observed when attacking the marked HDR images with additive Gaussian noise.

\section{Conclusions}

HDR images represent valuable content, whose intellectual property needs to be protected by means of proper digital techniques. To this aim, a blind multi-bit watermarking method for HDR images has been presented in this paper. The proposed approach hides information in areas closely associated to image contours, thanks to the properties of the considered RDCT transform. The selected embedding domain guarantees proper imperceptibility of the inserted marks, while providing the means for blindly extracting the embedded information without the need of the original image. An extensive set of experimental tests, conducted on 15 different HDR images, has been conducted to estimate the performance of the proposed method. The HDR-VDP-2 metric is employed to evaluate the perceptibility of the performed modifications, while six different TMOs have been employed to evaluate the possibility of retrieving the embedded data from LDR images generated from the marked HDR ones. The effects of signal processing attacks such as Gaussian additive noise and filtering have been also evaluated. The obtained results testify the effectiveness of the proposed approach, also showing the trade-off relationships between imperceptibility, robustness, and capacity when varying the parameters determining the employed system configurations.

Table 9 Robustness against malicious signal processing attacks, expressed in terms of BER (in \%). Results averaged over the considered images

\begin{tabular}{|c|c|c|c|c|c|c|c|c|}
\hline & & \multirow{3}{*}{ HDR } & \multicolumn{6}{|c|}{ TMO } \\
\hline & & & iCAM06 & tonemap & Reinhard & Drago & Tumblin & Durand \\
\hline & & & [25] & [26] & [27] & {$[28]$} & [29] & {$[20]$} \\
\hline \multirow{2}{*}{$\begin{array}{l}\text { Gaussian } \\
\text { noise }\end{array}$} & Proposed method & 28.43 & 30.59 & 34.78 & 30.13 & 34.43 & 32.24 & 35.42 \\
\hline & Wu et al. [18] & 31.07 & 31.10 & 30.69 & 30.79 & 30.88 & 31.02 & 31.08 \\
\hline \multirow{2}{*}{$\begin{array}{l}\text { Gaussian } \\
\text { blur }\end{array}$} & Proposed method & 11.59 & 22.31 & 29.25 & 17.82 & 23.22 & 22.28 & 23.92 \\
\hline & Wu et al. [18] & 32.97 & 32.73 & 33.24 & 33.12 & 33.29 & 33.83 & 33.56 \\
\hline
\end{tabular}




\section{Competing interests}

The authors declare that they have no competing interests.

\section{Received: 7 May 2015 Accepted: 6 December 2015}

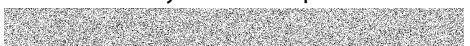

\section{References}

1. F Banterle, A Artusi, K Debattista, A Chalmers, Advanced High Dynamic Range Imaging: Theory and Practice. (AK Peters (CRC Press), Natick, MA, USA, 2011)

2. JJ McCann, A Rizzi, The Art and Science of HDR Imaging. (Wiley, USA, 2011)

3. F Barni, F Bartolini, Watermarking Systems Engineering: Enabling Digital Assets Security and Other Applications. (CRC Press, USA, 2004)

4. IJ Cox, M Miller, JA Bloom, J Fridrich, T Kalker, Digital Watermarking and Steganography. (Morgan Kaufmann, USA, 2008)

5. J Fridrich, Steganography in Digital Media: Principles, Algorithms, and Applications. (Cambridge University Press, USA, 2009)

6. E Maiorana, V Solachidis, P Campisi, in IEEE International Symposium on Image and Signal Processing and Analysis. Robust multi-bit watermarking for HDR images in the Radon-DCT domain (IEEE, Trieste, Italy, 2013)

7. XXue, T Jinno, J Xin, M Okuda, Watermarking for HDR image robust to tone mapping. IEICE Trans. Fundam. Electron. Commun. Comput. Sci. 94(11), 2334-2341 (2011)

8. F Guerrini, M Okuda, N Adami, R Leonardi, High dynamic range image watermarking robust against tone-mapping operators. IEEE Trans. Inf. Forensics Sec. 6(2), 283-295 (2011)

9. B Chen, GW Wornell, Quantization index modulation: a class of provably good methods for digital watermarking and information embedding. IEEE Trans. Inf. Theory. 47(4), 1423-1443 (2001)

10. R Mantiuk, K Myszkowski, H-P Seidel, in IEEE Internation Conference on Systems, Man and Cybernetics. Visible difference predictor for high dynamic range images (IEEE, The Hague, Netherlands, 2004)

11. F Autrusseau, D Goudia, in 20th IEEE International Conference on Image Processing (ICIP). Non linear hybrid watermarking for high dynamic range images (IEEE, Melbourne, Australia, 2013)

12. V Solachidis, E Maiorana, P Campisi, F Banterle, in IEEE International Conference on Digital Signal Processing (DSP). HDR image watermarking based on bracketing decomposition (IEEE, Santorini, Greece, 2013)

13. R Mantiuk, KJ Kim, AG Rempel, W Heidrich, HDR-VDP-2: a calibrated visual metric for visibility and quality predictions in all luminance conditions. ACM Trans. Graph. 30(4), 1-14 (2011)

14. Y-M Cheng, C-M Wang, A novel approach to steganography in high-dynamic-range images. IEEE Multimedia. 16(3), 70-80 (2009)

15. MT Li, NC Huang, CM Wang, A data hiding scheme for high dynamic range images. International Journal of Innovative Computing Information and Control (IJICIC). 7(5A), 2021-2035 (2011)

16. C-M Yu, K-C Wu, C-M Wang, A distortion-free data hiding scheme for high dynamic range images. Displays. 32(5), 225-236 (2011)

17. C Rattanacharuchinda, TAmornraksa, in International Conference on Electrical Engineering/Electronics, Computer, Telecommunications and Information Technology (ECTI-CON). Image watermarking for high dynamic range images (IEEE, Hua Hin, Thailand, 2012)

18. J-L Wu, in Watermarking - Volume 2. Robust watermarking framework for high dynamic range images against tone-mapping attacks (InTech, USA, 2012), pp. 229-242

19. V Solachidis, E Maiorana, P Campisi, in Proc. SPIE 8655, Image Processing: Algorithms and Systems XI, 865505. HDR image multi-bit watermarking using bilateral-filtering-based masking (SPIE, San Francisco, USA, 2013)

20. F Durand, J Dorsey, Fast bilateral filtering for the display of high-dynamic-range images. ACM Trans. Graph. 21(3), 257-266 (2002)

21. Z Farbman, R Fattal, D Lischinski, R Szeliski, Edge-preserving decompositions for multi-scale tone and detail manipulation. ACM Trans. Graph. 27(3), 1-10 (2008)

22. E Maiorana, P Campisi, A Neri, in IEEE Biometrics Symposium. Biometric signature authentication using Radon transform-based watermarking techniques (IEEE, Baltimora, USA, 2007)

23. S Mallat, $A$ Wavelet Tour of Signal Processing: The Sparse Way. (Accademic press, Waltham, MA, USA, 2008)

24. MN Do, M Vetterli, The finite ridgelet transform for image representation. IEEE Trans. Image Process. 12(1), 16-28 (2003)
25. J Kuang, GM Johnson, MD Fairchild, iCAM06: a refined image appearance model for HDR image rendering. J. Vis. Commun. Image Represent. 18(5), 406-414 (2007)

26. F Drago, K Myszkowski, T Annen, N Chiba, Adaptive logarithmic mapping for displaying high contrast scenes. EUROGRAPHICS. 22(3), 419-426 (2003)

27. E Reinhard, M Stark, P Shirley, J Ferwerda, Photographic tone reproduction for digital images. ACM Trans. Graph. 21(3), 267-276 (2002)

28. F Drago, K Myszkowski, T Annen, N Chiba, Adaptive logarithmic mapping for displaying high contrast scenes. 22(3), 419-426 (2003)

29. J Tumblin, H Rushmeier, Tone reproduction for realistic images. IEEE Comput. Graph. Appl. 13(6), 42-48 (1993)

\section{Submit your manuscript to a SpringerOpen ${ }^{\circ}$ journal and benefit from:}

- Convenient online submission

Rigorous peer review

- Immediate publication on acceptance

- Open access: articles freely available online

- High visibility within the field

- Retaining the copyright to your article

Submit your next manuscript at $\boldsymbol{\nabla}$ springeropen.com 